\section{DCs out of control}

The smooth running of all complex systems depends on precise regulation - even small defects in central control mechanisms can cause severe, system-wide problems. Systemic lupus erythematosus (SLE) is a chronic autoimmune disease involving both $\mathrm{T}$ - and $\mathrm{B}$-cell responses to ubiquitous self-antigens, which suggests that fundamental immune regulation is disrupted. A recent report in Science indicates that aberrations in dendritic cell (DC) homeostasis might be key to SLE pathogenesis.

In SLE, autoreactive antibodies cause inflammation in multiple organs, including the skin, joints, kidneys and central nervous system, but the aetiology of this disease is poorly defined. As DCs are the main regulators of innate and adaptive immunity, Patrick Blanco and co-workers, at the Baylor Institute for Immunology Research in Dallas, Texas, asked whether DC function is altered in SLE.

Two developmentally distinct DC subsets can be found in human blood - myeloid DCs (mDCs) and plasmacytoid DCs (pDCs). The authors show that mDCs, pDCs and monocytes (the precursors of mDCs) are all reduced in SLE blood, indicating that DC homeostasis is disrupted.

Interestingly, monocytes from SLE blood, unlike normal monocytes, are potent activators of naive T cells in vitro - a defining characteristic of DCs. Furthermore, when stimulated with SLE serum, normal monocytes develop DC-like morphology, phenotype and function, including the ability to engulf apoptotic cells and present the associated antigens to $\mathrm{CD} 4^{+}$ $\mathrm{T}$ cells. These results indicate that in SLE there might be mass differentiation of monocytes into DCs. The exodus of activated DCs into tissues could account for their reduced numbers in SLE blood.

What, then, is the factor in SLE blood that mediates these effects? Increased levels of CD40 ligand (CD154) and interferon- $\alpha$ (IFN- $\alpha$ ) are found in SLE blood, both of which have potent DC-activating effects. However, only neutralizing antibodies against IFN- $\alpha$ were found to abrogate the DC-stimulating activity of SLE serum - antibodies against CD154, interleukin-4 (IL-4) and granulocyte-macrophage colony-stimulating factor (GM-CSF; another DC activator) had no effect. Consistent with a role for IFN- $\alpha$, the DC-stimulating capacity of SLE serum was found to correlate with the levels of this cytokine.

But what is the source of IFN- $\alpha$ in SLE blood? pDCs were recently identified as the principal secretors of IFN- $\alpha$ in normal blood, but their numbers are drastically reduced in SLE. Furthermore, pDC depletion from SLE peripheral blood mononuclear cells results in only a partial reduction in virally induced IFN- $\alpha$ production. So there is an alternative source of IFN- $\alpha$ in SLE blood.

The authors propose that increased IFN- $\alpha$ in SLE drives a feedback loop of immune dysregulation. Blood monocytes activated by IFN- $\alpha$ become mature DCs, which take up apoptotic cells and activate autoreactive $\mathrm{CD} 4^{+} \mathrm{T}$ cells. The T cells then provide 'help' for the production of autoantibodies, which leads to the formation of immune complexes that cause organ damage. By interacting with $\mathrm{Fc}$ receptors on DCs and other immune cells, immune complexes might, in turn, sustain IFN- $\alpha$ production.

Although this model raises many questions, it identifies IFN- $\alpha$ as a potential therapeutic target in SLE. If IFN- $\alpha$ is central to SLE pathogenesis then, just as anti-tumour-necrosis factor has proved to be an effective therapy for rheumatoid arthritis, anti-IFN- $\alpha$ might halt the cycle of autoimmunity in SLE, and bring relief for patients.

D) References and links

Jennifer Bell

ORIGINAL RESEARCH PAPER Blanco, P. et al. Induction of dendritic cell differentiation by IFN- $\alpha$ in systemic lupus erythematosus. Science 294, 1540-1543 (2001) FURTHER READING Shlomchik, M.J. et al. From T to B and back again: positive feedback in system autoimmune disease. Nature Rev. Immunol. 1, 147-153 (2001)

\section{TNF SIGNALLING}

\section{Focusing on TRAF1}

Signalling by the tumour-necrosis factor (TNF) superfamily involves the TRAF (TNF receptor-associated factor) proteins that activate the NF- $\kappa B$ and AP-1 transcription factors. TRAF1 is unique in that it lacks a RING finger domain, and, rather than being ubiquitously expressed like other TRAF molecules, its expression is restricted to the spleen, lung and testis. Reporting in Immunity, Tsitsikov and colleagues show that Traf1 is a negative regulator of TNF signalling. Traf1-deficient mice developed normally and had no defects in B- or T-cell development. When immunized with ovalbumin, antibody responses developed as normal, in contrast to results from other Traf-deficient mice. To assess T-cell proliferative responses, $\mathrm{Traf1}^{-1-} \mathrm{T}$ cells stimulated with anti-CD3 antibody in vitro were compared with wild-type T cells. The Traf1 ${ }^{-1-} \mathrm{T}$ cells proliferated to a greater degree than wild-type $\mathrm{T}$ cells. This excess proliferation did not seem to involve interleukin-2 (IL-2) or co-stimulation via CD28, as increased expression of CD25 (the IL-2 receptor $\alpha$-chain) or IL-2 production in Trafl $1^{-1-}$ cells was not observed, and the blockade of CD28 signalling or addition of IL-2 did not block the response.

So, what is the explanation for the excessive responsiveness of the Trafl $1^{-1-} \mathrm{T}$ cells? When $\mathrm{T}$ cells were preactivated by anti-CD3 antibody for 3 days, the Traf1 ${ }^{-1-}$ cells proliferated in response to TNF, but the wild-type cells did not. TNF receptor 2 (Tnfr2) is expressed on activated T cells, and anti-Tnfr2 antibodies specifically blocked the proliferative response of the Trafl $^{-1-}$ cells. These results indicate that Traf1 negatively regulates Tnfr2-mediated proliferative signals in $\mathrm{T}$ cells.

The authors next investigated the downstream signalling events following TNF stimulation of Traf1 ${ }^{--}-\mathrm{T}$ cells. Activation of both the NF- $\mathrm{KB}$ and AP-1 pathways was enhanced in the Traf1-deficient cells following TNF stimulation. In the NF- $\kappa B$ pathway, TNF stimulation resulted in increased inhibitory $\mathrm{\kappa B}$ kinase (IKK) activity, enhanced translocation of NF- $\kappa \mathrm{B}$ to the nucleus and increased expression of genes that are regulated by NF- $\mathrm{\kappa B}$. TNF stimulation of $\mathrm{Trafl}^{1^{--}} \mathrm{T}$ cells also resulted in increased phosphorylation of c-Jun $\mathrm{N}$-terminal kinase (JNK) signalling molecules and nuclear AP-1 binding activity.

Collectively, these results indicate that Traf 1 is a negative regulator of proliferative signals delivered by Tnfr 2 . Trafl could mediate this inhibition in four ways - competition with Traf2 for binding to Tnfr2, generation of an inactive heterodimer with Traf2, regulation of molecules that are involved in Traf2 signalling (other than Traf2), or recruitment of molecules that negatively regulate TNF signalling. Further work will be required to elucidate the precise mechanisms of Traf 1 regulation of Tnfr2 signalling, but the importance of TNF molecules in many immune-mediated diseases means that this will be a worthwhile effort.

Elaine Bell

(i)) References and links ORIGINAL RESEARCH PAPER Tsitsikov, E. N. et al. TRAF1 is a negative regulator of TNF signaling: enhanced TNF signaling in TRAF1-deficient mice. Immunity $\mathbf{1 5}$ 647-657 (2001) 